COMMENT. In the differential diagnosis of dystonia, a CT scan showing calcification in the basal ganglia lead to tests for hypoparathyroidism whereas an MRI may be helpful in the diagnosis of Hallervorden-Spatz disease. Dystonia and striking MRI abnormalities in the globus pallidus ("eyeof-the-tiger" sign) are described in 2 patients with Hallervorden-spatz syndrome (Sethi KD et al. Ann Neurol Nov 1988;24:692). One patient developed arching of the body backward and a diagnosis of dystonia at 14 years. She slowly deteriorated and at age 20 had progressive difficulty with mouth closure and at 32 years, frequent falling. CT scan of the head was normal whereas the MRI T2-weighted images showed a low-signal area surrounding a relatively circumscribed region of high signal in the globus pallidus. The 'eye-of-the-tiger' sign is an appropriate name for this abnormality. The second patient, a 20-year-old woman, had a 1-year history of progressive difficulty with night vision and repeated forceful eye closure aggravated by sudden noise and attempted eye opening. The neurological findings were frequent blepharospasm, repetitive slow tongue protrusion, and tapetoretinal degeneration. Slow deterioration occurred over the next 2 years and reexamination showed facial grimacing, severe blepharospasm, tongue protrusion, resting tremor of hands, tongue, and jaw, and cogwheel rigidity of arms." CT scan was normal and the MRI showed the "eye-oftiger" sign in the globus pallidus.

\title{
CNS INFECTIONS
}

\section{BACTERIAL MENIGITIS AND DEAFNESS}

Dexamethasone (.15 $\mathrm{mg} / \mathrm{kg} / \mathrm{b} w \mathrm{qt}$ q $6 \mathrm{hr}$ for 4 days) was considered beneficial in the treatment of infants and children with bacterial meningitis, particularly in preventing deafness, in two double blind, placebo-controlled trials involving 200 patients treated in the Dept of Pediatrics, University of Texas at Southwestern Medical Center, Dallas, TX. As compared to 98 patients receiving placebo, 102 treated with dexamethasone became afebrile earlier ( 1.6 vs 5 days; $P<.001)$ and were less likely to acquire bilateral sensorineural hearing loss (15.5 vs $3.3 \% ; \mathrm{P}<.01)$. Twelve patients in the 2 placebo groups $(14 \%)$ had severe bilateral loss as compared with $1(1 \%)$ ) in the 2 dexamethasone groups $(\mathrm{P}<.001)$. (Lebel MH et al. Dexamethasone therapy for bacterial meningitis. Results of 2 double-blind,

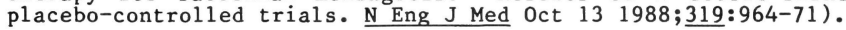

COMMENT. An editorial in the same issue (Smith AL. Neurological sequelae of meningitis $N$ Engl $J$ Med $1988 ; 319: 1012$ ) applauds the investigators for undertaking a difficult and complex study but notes that enthusiasm for the findings is dampened by the lack of follow-up of all patients enrolled in the study. The patients may have been restudied too early to detect improvement in auditory acuity 
of controls, and the dexamethasone group may have been less severely ill than the controls. An assessment of higher cortical function one year after discharge revealed no significant difference in treatment and control groups. Dexamethasone in the treatment of a severely ill child with meningitis may be recommended but a confirmatory study documenting safety is advised to determine its necessity in mild cases. Dexamethasone may save the hearing but worsen cerebal cortical function by ischemic injury and may induce gastrointestinal bleeding.

\section{INTRACRANIAL TUBERCULOMA}

In a report from the Dept of Pediatrics, All India Inst of Applied Sciences, New Delhi, India, intracranial tuberculoma was found in $20(24 \%)$ of 83 patients with partial seizures complicated by increased intracranial pressure, systemic tuberculosis or focal neurologic deficit and in $12(22 \%)$ of 55 patients with tuberculous meningitis. CT lesions consisting of ring enhancement, discs, and irregular coalescing masses with edema regressed within 12 weeks of starting medical therapy. Surgical excision was not required. Medical management was advocated, with surgery limited to drug treatment failures. (Bagga A et al. Intracranial tuberculoma. Evaluation and treatment. Clin Pediat Oct 1988;27:487-490).

COMMENT. Tuberculoma mimics other space-occupying lesions and in India its reported prevalence has ranged from 4 to $40 \%$ of intracranial tumors. Effective chemotherapy and CT monitoring of treatment response have minimized the role of surgery.

\section{INTRACRANIAL TUMORS}

RADIATION-INDUCED TUMORS

The relation between radiotherapy to the head and neck for tinea capitis childhood and the later development of tumors of the brain and nervous system have been investigated in 10,834 patients treated between 1948 and 1960 in Israel and the results evaluated at the Radiation Epidemiology Branch, National Cancer Institute, Bethesda, MD. Neural tumors developed in 73 patients, 60 in irradiated subjects, 8 among general population controls, and 5 among sibling controls. The increase in incidence among those irradiated was 7 times that of controls. The relative risk of all head and neck situated neural tumors among irradiated subjects was 8.4 . Increased relative risks were greater for benign nerve-sheath tumors $(18.8 ; n=25)$ than for meningiomas $(9.5 ; n=19)$ and gliomas $(2.6 ; n=7)$. A strong doseresponse relation was shown, the risk approaching 20 after doses of $2.5 \mathrm{~Gy}$. Radiation doses to the head and neck in childhood on the order of 1 to 2 GY significantly increased the risk of neural tumors in those areas. (Ron $E$ et al Tumors of the brain and nervous system after radiotherapy in childhood. $N$ Engl J Med Oct 20 1988; 319:1033-9). 